\title{
Isolation of Mallory bodies and an attempt to demonstrate cell mediated immunity to Mallory body isolate in patients with alcoholic liver disease
}

\author{
C GLUUD, F HARDT, J ALDERSHVILE, P CHRISTOFFERSEN, H LYON, \\ JO NIELSEN
}

From the Department of Medicine, Division of Hepatology, and Department of Pathology, Hvidovre Hospital, and Second Department of Medicine, Kommunehospitalet, University of Copenhagen, Denmark

SUMMARY Mallory bodies were isolated from necropsy livers from patients with alcoholic hepatitis with and without cirrhosis with a Ficoll viscosity barrier. The purity of Mallory bodies in the isolate varied between 70 and $90 \%$, estimated by counting Mallory bodies and non-Mallory body structures in haematoxylin-eosin stained smears. Electron microscopy confirmed the presence of Mallory bodies in the isolates. The Mallory body isolate was used as antigen in the agarose leucocyte migration inhibition test in order to test the cell-mediated immunity. No significant difference in leucocyte migration was found between controls and patients with alcoholic hepatitis, alcoholic steatosis, alcoholic cirrhosis and miscellaneous liver diseases.

Mallory bodies (MBs), or alcoholic hyalin, are an important histological hallmark of alcoholic hepatitis. ${ }^{1}$ Since French et al. ${ }^{2}$ described the isolation of MBs a number of investigations have dealt with the isolation and characterisation of MBs. $^{3-6}$ These studies indicate that MBs are composed of branching, tubular filaments, possibly derived from intermediate filaments. ${ }^{5} 6 \mathrm{MBs}$ seem to be composed of five or more polypeptides ${ }^{4-7}$ and contain carbohydrate. ${ }^{6} 7$

Leevy and collaborators have reported both cell mediated $^{8-12}$ and humoral ${ }^{13-15}$ immunity to isolated MBs in patients with alcoholic hepatitis. These findings generated the hypothesis that MBs could be a neoantigen induced by ethanol. This antigen should provoke both cellular and humoral immune response, which could lead to the development of alcoholic hepatitis and progression from alcoholic hepatitis to cirrhosis. ${ }^{12} 14$ These findings, however, have not been reproduced by other groups. ${ }^{16-19}$

In the present study MBs were isolated from human liver obtained at necropsy and used as antigen in the agarose leucocyte migration inhibition test in order to evaluate the cell mediated immune response in patients with alcoholic liver disease.

Accepted for publication 11 March 1981
Material and methods

ISOLATION OF MBS

A modification of a procedure described by $\mathrm{HM}$ Tinberg and SW French (personal communication, 1977) was used for the isolation of MBs. Livers were removed at necropsy (within 16 hours of death) from chronic alcoholics dying with alcoholic hepatitis with and without cirrhosis. The presence of MBs was confirmed on paraffin sections stained with haematoxylin and eosin. The livers were sliced and stored at $-70^{\circ} \mathrm{C}$ in isopentane until use.

Frozen livers $(250 \mathrm{~g})$ were partially thawed, cut into smaller pieces, and ground at top speed in a Waring Blendor for $1 \frac{1}{2}$ minutes in four volumes of isolation medium ${ }^{6}$ at $4^{\circ} \mathrm{C}$. Fine grade glass wool (Pyrex Wool, Corning Glass Works) was added to the homogenate in order to absorb collagen. The homogenate was separated from the soaked glass wool by squeezing it through surgical gauze followed by filtration on a Buchner funnel lined with three layers of glass wool and one layer of surgical gauze. The glass wool was repeatedly washed with isolation medium and the rinses added to the Buchner funnel and additional isolation medium was used to wash the filter. At this point the original volume was doubled. A considerable amount of contamination with 
glass wool was noticed. Because of this, the homogenate was filtered through two layers of nylon mesh (pore size 40 and $10 \mu \mathrm{m}$ ) on a Buchner funnel. The filtrate was centrifuged at $365 \mathrm{~g}$ for $10 \mathrm{~min}$ and the supernatant was sucked off leaving a yellow-greenwhite pellet. The latter was resuspended in isolation medium by vigorous shaking and centrifugation was repeated 2-3 times until the supernatant was clear. The pellet was then digested ${ }^{5}$ at $37^{\circ} \mathrm{C}$ overnight in $0.05 \mathrm{~mol} / 1$ Tris buffer $\mathrm{pH} 7.5$ containing $0.1 \%$ DNAase, $0.1 \%$ RNAase, $0.1 \%$ collagenase (Worthington Biochemical Corp), $0.36 \mathrm{mmol} / 1 \mathrm{CaCl}_{2}, 0 \cdot 1$ $\mathrm{mmol} / 1 \mathrm{MgSO}_{4}$, and $0.1 \%$ Thiomersal. Then the suspension was washed three times in ice-cold deionised water. The pellet was incubated in $5 \%$ deoxycholate at $37^{\circ} \mathrm{C}$ overnight, washed three times in ice-cold deionised water and resuspended in $0.25 \mathrm{~mol} / 1$ sucrose using an MSE homogenizer (top speed, $2 \mathrm{~min}$ ) to a total volume of $30 \mathrm{ml}$. Five $\mathrm{ml}$ were layered on $30 \mathrm{ml}$ Ficoll $400(40 \% \mathrm{wt} / \mathrm{wt})$ and centrifuged for $65 \mathrm{~min}$ at $20000 \mathrm{rpm}(48200 \mathrm{~g})$ at $4^{\circ} \mathrm{C}$ (Sorwall Super Speed Refrigerated Centrifuge). The pellet was washed in 20 volumes of deionised water and stored at $-20^{\circ} \mathrm{C}$ in $0.01 \%$ acetic acid until use.

\section{Light microscopy}

During the isolation procedure smears of fractions and final pellets were stained with haematoxylin and eosin and examined for presence and purity of MBs.

\section{Electron microscopy}

Isolates of MBs were fixed in 3\% glutaraldehyde in $0.1 \mathrm{~mol} / 1$ sodium phosphate buffer $(\mathrm{pH} 7.4)$ for two hours. After fixation the isolate was washed in phosphate buffer and embedded in Epon. Thick sections $(1 \mu \mathrm{m})$ were stained by toluidine blue. Thin sections $(500 \AA)$ were stained in uranyl acetate and lead citrate and examined in a Jeol 100 electron microscope.

\section{Solvents}

In order to solubilise MBs for application in the leucocyte migration inhibition test the following solvents were tested for their ability to dissolve MBs and their effect on spontaneous leucocyte migration index: (a) Guanidine buffer (guanidine $\mathbf{H C l} 2$ $\mathrm{mol} / \mathrm{l}$, mercaptoethanol $50 \mathrm{mmol} / \mathrm{l}$, disodiumphosphate $10.5 \mathrm{mmol} / 1$, pH 7.5);12 (b) guanidine $\mathrm{HCl} 6$ $\mathrm{mol} / \mathrm{l}$; (c) $\mathrm{NaOH} 1 \mathrm{~mol} / 1$ and $40 \mathrm{mmol} / \mathrm{l}$; (d) sodium dodecyl sulphate (SDS) $1 \%$.

Preparation of $M B$ isolate for the leucocyte migration inhibition test

MB isolate was dissolved in SDS $1 \%$ and dialysed overnight against $1200 \mathrm{ml} \mathrm{NaOH} 40 \mathrm{mmol} / \mathrm{l}$. The protein concentration was $1 \cdot 8 \mathrm{mg} / \mathrm{ml}$. This material was used as antigen in the leucocyte migration inhibition test after dilution with phosphate buffer to a concentration giving rise to no inhibition of migration by normal controls (see Results).

\section{Leucocyte migration inhibition test}

The agarose leucocyte migration inhibition test was used as described in detail elsewhere. ${ }^{20}$ In summary, leucocytes were prepared from controls and patients to a final concentration of $2 \cdot 2 \times 10^{8}$ leucocytes per $\mathrm{ml}$. $2 \times 10^{8}$ leucocytes were incubated with $10 \mu \mathrm{l} \mathrm{MB}$ isolate prepared as described above. As control, $2 \times 10^{8}$ leucocytes were incubated in phosphate buffer without MB isolate. Seven $\mu$ l of the leucocyteantigen mixture were placed in three wells, and the control mixture was placed in two other wells in the agarose plate. The plates were incubated for $24 \mathrm{~h}$ at $37^{\circ} \mathrm{C}$ in a $2 \% \mathrm{CO}_{2}$ in air saturated with water vapour. The migration area was read under a projection microscope and migration index was calculated

$$
M I=\frac{R a^{2}-r^{2}}{R c^{2}-r^{2}}
$$

where $\mathrm{Ra}$ is the radius of migration with antigen, $\mathrm{Rc}$ migration in the control, and $\mathrm{r}$ radius of the wells.

\section{PATIENTS}

Twenty-nine patients and 16 healthy staff members were included. The median age of patients was 52 years (range 17-77 years) and seven were women. A liver biopsy was performed in 27 of the patients, and based on histological, clinical and biochemical findings, the patients were classified into the following groups:

Alcoholic hepatitis $(\mathrm{n}=11)$. All had abused alcohol and the diagnosis was confirmed by biopsy $(n=9)$ and/or by the classical symptoms of fever, jaundice, leucocytosis, and upper quadrant pain. ${ }^{1}$ Two patients had no biopsy due to low coagulation factors. Seven of the nine biopsies also showed cirrhosis.

Alcoholic cirrhosis $(\mathrm{n}=6)$. All had abused alcohol and had a biopsy showing cirrhosis compatible with an alcoholic aetiology.

Alcoholic steatosis $(\mathrm{n}=4)$. All had abused alcohol and biopsies showed steatosis of the liver (and fibrosis in one).

Miscellaneous liver diseases $(\mathrm{n}=8)$. According to clinical, serological and histological criteria this group comprised one patient with Gilbert's syndrome, three with acute viral hepatitis, two with cryptogenic 
Fig. 1 Light microscopy of Mallory body isolate from human liver stained with haematoxylin and eosin. The preparation consists mainly of Mallory bodies. The small particles are glass wool $\times 250$.

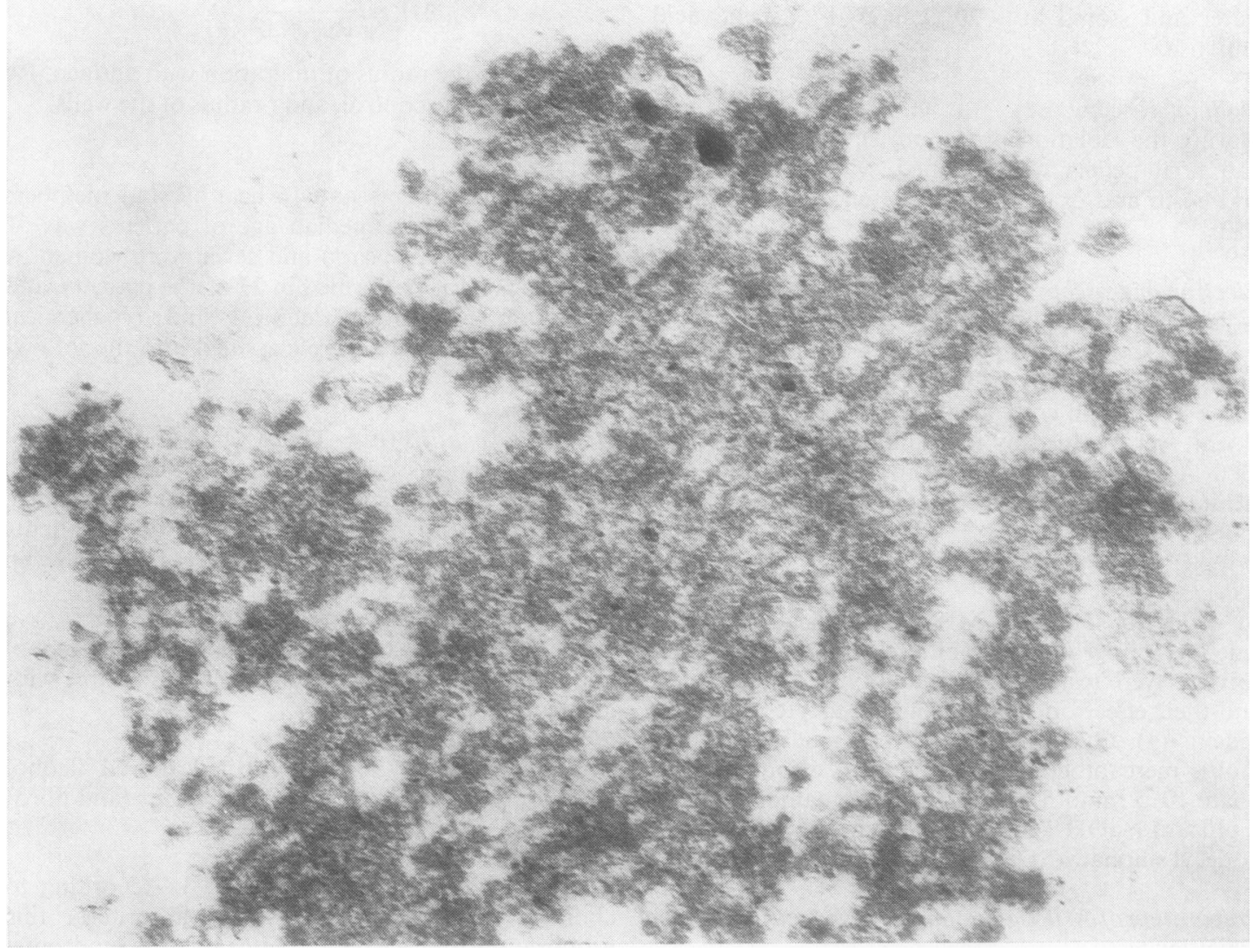

Fig. 2 Electron microscopy of Mallory body isolate. The characteristic filamentous structure is well preserved. Uranyl acetate and lead citrate $\times 10000$. 
cirrhosis, one with chronic active hepatitis and cirrhosis, and one with cirrhosis and $\alpha_{1}$-antitrypsin deficiency.

\section{Results}

ISOLATION OF MBS

Counting MB and non-MB structures in haematoxylin and eosin stained smears (Fig. 1) the purity of MBs in the isolates varied between 70 and $90 \%$. Most of the contaminating material consisted of nuclei and unidentifiable debris. Introducing a nylon mesh in the isolation procedure reduced the contamination of glass wool, but not totally. Yields of protein in the MB isolate averaged 2-5 $\mathrm{mg}$ protein per $100 \mathrm{~g}$ of liver wet weight.

Electron microscopy of the isolates confirmed the presence of MBs (Figs 2 and 3). The characteristic filamentous ultrastructure and tubular appearance was seen and did not differ from that of MBs in freshly obtained liver biopsy specimens. According to the classification of Yokoo et al., ${ }^{21}$ both type II and type III MBs were identified, but no type I MBs were observed.

\section{SOLUBILISATION OF MBS AND TOXICITY OF SOLVENTS}

Varying and usually small amounts of the MB isolate could be solubilised using the guanidine buffer, guanidine $\mathrm{HCl} 6 \mathrm{~mol} / \mathrm{l}$, and $\mathrm{NaOH}$. Using SDS $1 \%$ about $90 \%$ of the MB isolate could be solubilised.

Evaluating the spontaneous effect of these solvents on leucocyte migration index of normal persons showed that guanidine buffer, guanidine $\mathrm{HCl} 6$ $\mathrm{mol} / \mathrm{l}$, SDS $1 \%$ and $\mathrm{NaOH} 1 \mathrm{~mol} / 1$ all were toxic and gave small migration indices $(<0 \cdot 30)$ compared to phosphate buffer. $\mathrm{NaOH}(40 \mathrm{mmol} / \mathrm{l})$ did not affect the migration index and diluting this concentration further did not increase the migration index. Diluting the guanidine buffer and guanidine $\mathrm{HCl}$ $6 \mathrm{~mol} / \mathrm{l} 10-20$ times and SDS $1 \% 200$ times abolished the inhibition of migration. MB isolate of high purity was hereafter dissolved in SDS $1 \%(5 \mathrm{ml})$ and dialysed against $1200 \mathrm{ml} \mathrm{NaOH} 40 \mathrm{mmol} / \mathrm{l}$ as described in Material and methods. This material was used for further studies.

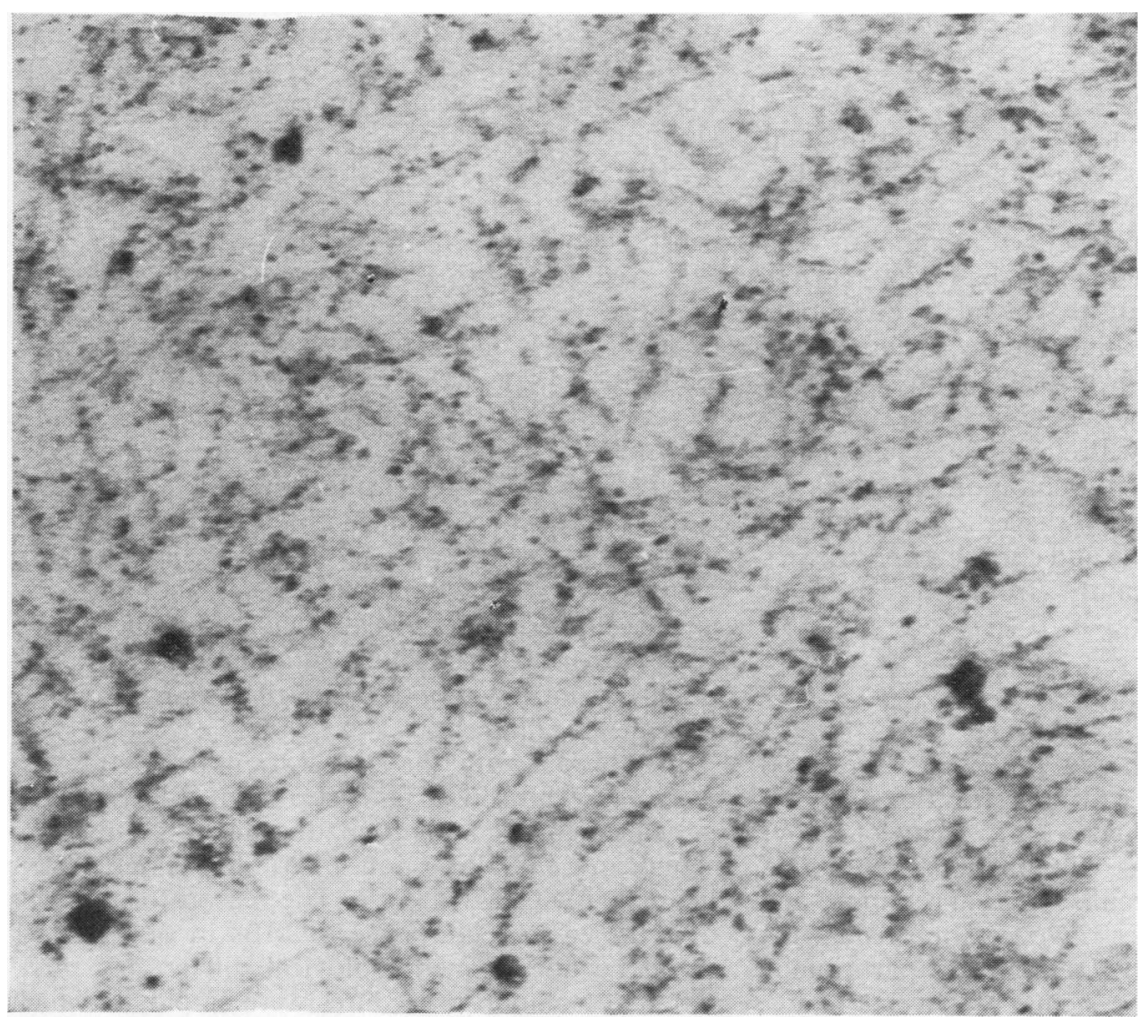

Fig. 3 Electron microscopy of Mallory body isolate. The filamentous nature is evident. Uranyl acetate and lead citrate $\times 99000$. 
LEUCOCYTE MIGRATION INHIBITION IN

CONTROLS AND PATIENTS

In Fig. 4 the effect of the dissolved MB isolate on the migration of leucocytes from control persons is shown. The antigen had to be diluted 40-60 times before no significant inhibition of migration occurred. A 40 times dilution (corresponding to a protein concentration of $0.045 \mathrm{mg} / \mathrm{ml}$ ) was used hereafter.

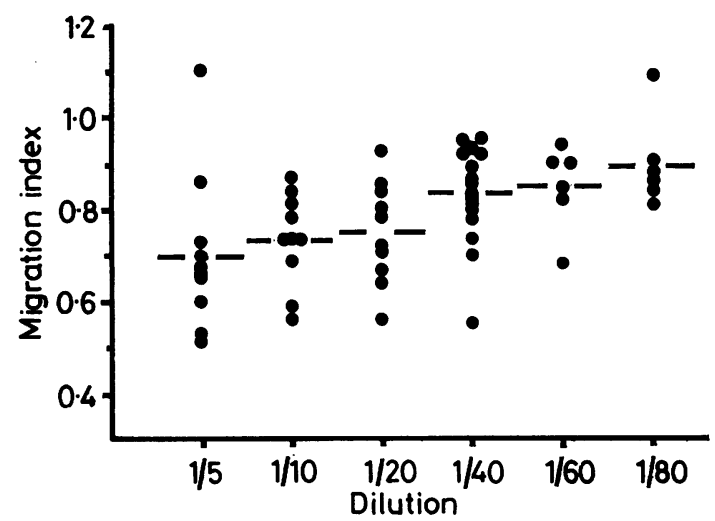

Fig. 4 Effect of Mallory body isolate on migration index of leucocytes from normal controls. Mallory body isolate was prepared as described in Material and methods. Broken horizontal line represents mean migration index.

In Fig. 5 the migration indices of controls and patients with liver diseases are shown. In 13 healthy controls the mean migration index was 0.85 ; a significant inhibition of leucocyte migration was defined as a migration index less than $0 \cdot 69$, corresponding to the mean minus two standard diviations. As shown no significant difference between normal controls and patients with alcoholic hepatitis, alcoholic cirrhosis and alcoholic steatosis was observed. Two patients with alcoholic hepatitis and one with steatosis showed inhibition of leucocyte migration according to the criteria outlined above. Patients with various acute and chronic liver diseases did not differ significantly from the previous mentioned groups.

\section{Discussion}

Using the method of Tinberg and French (personal communication, 1977), with modifications as described, MBs were isolated with a purity of about $80 \%$. This is in accordance with previously published results. ${ }^{3} 7 \mathrm{MBs}$ have been isolated in semipure and pure fractions by other investigators using the method of Okamura et al. employing partitioning in a two phase polymer system. ${ }^{4} 714$ In our hands,

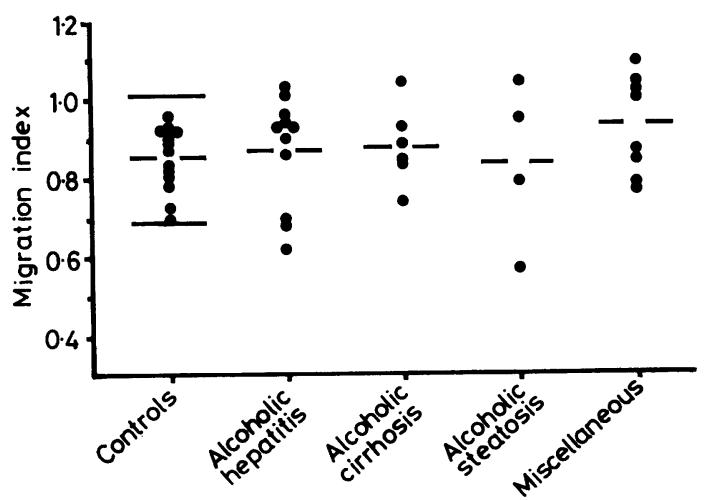

Fig. 5 Migration index of leucocytes from normal controls, patients with alcoholic liver disease and miscellaneous liver diseases after addition of Mallory body isolate. Broken horizontal line represents mean migration index and solid horizontal line represents $2 S D$.

however, only small and contaminated preparations of MBs could be obtained by this method. Similar difficulties have recently been reported by others. ${ }^{6}$

The MBs isolated in the present study appear very much as in situ with a characteristic rope-like appearance when seen by light microscopy. Electron microscopy confirmed the presence of $\mathrm{MBs}$, and both type II and III MBs were identified according to the classification of Yokoo et al. ${ }^{21}$ No type I MBs were identified, which is in accordance with pre-" vious observations. ${ }^{6}$

MBs are composed of branching filaments, and are thought to be derived from a normal protein constituent of the liver cell cytosol. Reports that MBs were related to actin ${ }^{22}$ have not been confirmed in more recent studies. ${ }^{523}$ The constituent that resembles MB filaments best is the intermediate filament. ${ }^{23}$ Like MBs they do not bind heavy meromycin, and intermediate filaments can absorb MB-specific antibody. ${ }^{5}$ Other investigators have stressed that MBs are related to-but not identical with-intermediate filaments. ${ }^{24}$ Antibodies towards neurofilaments, known to bind to intermediate filaments, did not bind to $\mathrm{MBs},{ }^{5}$ and antibody against human glial fibrillary acidic protein did not react with isolated MBs (C Gluud and E Bock, unpublished observations). The glial filament is an intermediate filament. ${ }^{25}$

In contrast with the observations of Zetterman et al., ${ }^{10} 12$ but in accordance with MacSween, ${ }^{18}$ patients with alcoholic hepatitis did not show any significant inhibition of migration index when incubated with MB isolate. The reason for the lack of cell-mediated immunity to MBs in our patients could be due to methodological differences. First, different isolation procedures were employed which 
may have led to differences in the antigenic composition. Secondly, MBs were solubilised in SDS followed by $\mathrm{NaOH}$ dialysis in the present study. Zetterman et al. ${ }^{12}$ used the guanidine buffer. MBs are difficult to solubilise $e^{323}$ and varying and rather small fractions of the MB isolate could be solubilised using this buffer. Further, the guanidine buffer is toxic to leucocytes when undiluted, an observation also made by MacSween. ${ }^{18}$ The guanidine buffer contains mercaptoethanol, which can convert non-adherent (macrophage-free) cells into a population with the functional immune properties of macrophages. Therefore, mercaptoethanol may affect leucocytes from patients with alcoholic hepatitis in an unpredictable way producing migration inhibition. ${ }^{26}$ Thirdly, Zetterman et al.12 used the capillary migration test, while in the present study the agarose leucocyte migration inhibition test was employed. This method is easier to reproduce and uses smaller amounts of antigen. MacSween noted that the leucocyte migration of patients with alcoholic hepatitis was reduced more after preincubation with MBs rather than by adding leucocytes directly to the culture chamber, although the results did not achieve statistical significance. ${ }^{18}$ The protein concentration used in our leucocyte migration test is comparable with that used in earlier studies. ${ }^{20}$

The pathogenetic role of MBs in the development of alcoholic liver diseases still seems to be controversial, and further studies to clarify the role of MBs and autoimmune mechanisms in the progression of alcoholic liver disease are needed.

\section{Addendum}

Since the preparation of this manuscript, Triggs et $a .^{27}$ have published results which showed migration inhibition to MBs in some patients with alcoholic hepatitis. Using the capillary migration test, they found only migration inhibition when using preincubation. As in the present paper Triggs et al. ${ }^{27}$ also reported a toxic effect for leucocytes of a number of solvents. They therefore used sonicated suspensions of MBs in saline as antigen material.

We thank Professor SW French, California, for his assistance concerning isolation of MBs. Thanks are expressed to Lena Hansen and Hanna Pedersen for skilful technical assistance. This work was supported by grants from the Danish Medical Research Council, the Danish Foundation for the Advancement of Medical Science and the Ebba Celinder Foundation.

\section{References}

${ }^{1}$ Leevy CM, Popper H, Sherlock S. Alcoholic hepatitis. In: Diseases of the liver and biliary tract. Standardization of nomenclature, diagnostic criteria and diagnostic methodology. Fogarty International Center Proceedings, No. 22. US Government Printing Office, 1976:6.

2 French SW, Ihrig TJ, Norum ML. A method of isolation of Mallory bodies in a purified fraction. Lab Invest 1972; 26:240-4.

${ }^{3}$ Okamura K, Harwood TR, Yokoo H. Isolation and amino acid analysis of Mallory bodies. Prep Biochem 1974;4: 331-40.

${ }^{4}$ Okamura K, Harwood TR, Yokoo H. Isolation and electrophoretic study on Mallory bodies from the livers of alcoholic cirrhosis. Lab Invest 1975;33:193-9.

${ }^{5}$ French SW, Sim JS, Caldwell MG. Thick microfilaments (intermediate filaments) and chronic alcohol ingestion. In: Popper $\mathrm{H}$, ed. Membrane alterations as basis of liver injury. Lancaster MTP Press. 1977: 311-25.

- Tinberg HM, Regan RJ, Geier EA, Petersen GE, French SW. Mallory bodies. Isolation of hepatocellular hyalin and electrophoretic resolution of polypeptide components. Lab Invest 1978;39:483-90.

${ }^{7}$ Luisada-Opper AV, Kanagasundaram N, Leevy CM. Chemical nature of alcoholic hyalin. Gastroenterology 1977;73:1374-6.

${ }^{8}$ Leevy CM, Zetterman R, Sorrell M, Luisada-Opper A. Immunologic reactivity in alcoholic hepatitis. Clinical Research (Abstract) 1973;21:723.

- Leevy CM, Chen T, Zetterman R. Alcoholic hepatitis, cirrhosis and immunologic reactivity. Ann NY Acad Sci 1975;252:106-15.

${ }^{10}$ Zetterman RK, Leevy CM. Immunologic reactivity and alcoholic liver disease. Bull NY Acad Med 1975;51: 533-44.

${ }^{11}$ Leevy CM, Chen T, Luisada-Opper A, Kanagasundaram N, Zetterman R. Liver disease of the alcoholic: Role of immunologic abnormalities in pathogenesis, recognition, and treatment. In: Popper H, Schaffner F, eds. Progress in liver disease Vol. 5. New York: Grune Stratton, 1976:516-30.

12 Zetterman RK, Luisada-Opper A, Leevy CM. Alcoholic hepatitis. Cell-mediated immunological response to alcoholic hyalin. Gastroenterology 1976;70:382-4.

${ }^{13}$ Chen T, Kanagasundaram N, Kakumu N, Luisada-Opper A, Leevy CM. Serum autoantibodies to alcoholic hyalin in alcoholic hepatitis. Gastroenterology (Abstract) 1975;69: 813.

${ }^{14}$ Kanagasundaram N, Kakumu S, Chen T. Leevy CM. Alcoholic hyalin antigen (AHAg) and antibody (AHAb) in alcoholic hepatitis. Gastroenterology 1977;73:1368-73.

${ }^{15}$ Kanagasundaram N, Leevy CM. The antigenic moiety in alcoholic hyalin $(\mathrm{AH})$ : Its identification and detection of AHAb by radioimmunoassay. Gastroenterology (Abstract) 1979;77:A20.

26 Junge U, Kehl A, Schober A, Winckler K. Immunological aspects of alcoholic hepatitis. Gastroenterology 1975; 75:165-6.

${ }^{17}$ Depew WT, Tong MJ, Tinberg H. Failure of purified alcoholic hyalin to induce lymphocyte transformation in acute alcoholic hepatitis. Gastroenterology (Abstract) 1978:75:960.

18 MacSween RNM. Immune responses to hyalin. In: Eddleston ALWF, Weber JCP, Williams R, eds. Immune reactions in liver disease. London: Pitman Medical, 1979: 215-9.

19 Kehl A, Schober A, Junge U, Winckler K. Solid-phase radioimmunoassay for detection of alcoholic hyalin antigen (AHAg) and antibody (anti-AH). Clin Exp Immunol 1981;43:215-21.

${ }^{20}$ Aldershvile J, Dietrichson O, Hardt F, Nielsen JO, Skinhøj P. Humoral and cell-mediated immunity to 
hepatitis B virus antigens in acute and chronic liver disease. Scand J Gastroenterol 1977;12:917-22.

${ }^{21}$ Yokoo H, Minick OT, Batti F, Kent G. Morphologic variants of alcoholic hyalin. Am J Pathol 1972;69:25-40.

22 Nenci I. Identification of actin-like proteins in alcoholic hyaline by immunofluorescence. Lab Invest 1975;32: 257-60.

${ }^{23}$ French SW, Davies PL. The Mallory body in the pathogenesis of alcoholic liver disease. In: Khanna JM, Kalant K, eds. Alcoholic liver pathology. Canada, Toronto: House of Lind, 1975:113-43.

" Franke WW, Denk H, Schmid E, Osborn M, Weber K. Ultrastructural, biochemical, and immunologic characterization of Mallory bodies in livers of griseofulvintreated mice. Lab Invest 1979; 40: 207-20.
25 Bock, E, Rasmussen S, Møller M, Ebbesen P. Demonstration of a protein immunochemically related to glial fibrillary acidic protein in human fibroblasts in culture. FEBS Lett 1977;83:212-6.

${ }^{26}$ Bull DM. Assessment of autoantigenic activity of hepatic tissue components. Gastroenterology 1976;71:164-5.

27 Triggs SM, Mills PR, MacSween RNM. Sensitisation to Mallory bodies (alcoholic hyalin) in alcoholic hepatitis. J Clin Pathol 1981;34:21-4.

Requests for reprints to: Christian Gluud, Department of Medicine, Division of Hepatology, Hvidovre Hospital, DK-2650 Copenhagen, Denmark. 